American Journal of Internal Medicine
2017; 5(4): $61-66$
http://www.sciencepublishinggroup.com/j/ajim
doi: 10.11648 /j.ajim.20170504.13
ISSN: $2330-4316$ (Print); ISSN: $2330-4324$ (Online)

\title{
Thrombosis and Cancer: Case Series of 25 Patients
}

\author{
Said Kaddouri ${ }^{1, ~}$, Zakaria Chahbi ${ }^{1}$, Ismail Essadi ${ }^{2}$, Hassan Qacif ${ }^{1}$, Mohamed Zyani ${ }^{1}$ \\ ${ }^{1}$ Department of Internal Medicine, IbnSina Military Hospital, Marrakesh, Morocco \\ ${ }^{2}$ Department of Medical Oncology, IbnSina Military Hospital, Marrakesh, Morocco
}

Email address:

Saidkad2004@yahoo.fr (S. Kaddouri)

${ }^{*}$ Corresponding author

\section{To cite this article:}

Said Kaddouri, Zakaria Chahbi, Ismail Essadi, Hassan Qacif, Mohamed Zyani. Thrombosis and Cancer: Case Series of 25 Patients. American Journal of Internal Medicine. Vol. 5, No. 4, 2017, pp. 61-66. doi: 10.11648/j.ajim.20170504.13

Received: May 3, 2017; Accepted: May 10, 2017; Published: July 6, 2017

\begin{abstract}
The objective of this paper is to describe the clinical and therapeutic characteristics of thrombosis associated with cancers in patients in charge of the internal medicine-oncology department of the Ibn Sina Military Hospital in Marrakech. This is a retrospective, descriptive and analytical study over a two-year period from January 2013 to December 2014 . Among 158 patients with cancer, we identified 25 with thrombosis confirmed by imaging. Characteristics of the population of origin, incidence, risk factors for venous thromboembolic disease (VTE), diagnostic means, distribution of thrombosis, location and histological type of cancer and therapeutic strategy were analyzed. The mean age of our patients was 55.89 years, with female predominance $(56 \%$ vs $44 \%)$. The incidence of venous thrombosis in our series is $15.8 \%(n=25)$ of which $76 \%(n=19)$ are isolated, $12 \%(n=3)$ are on central venous catheter $(\mathrm{CVC})$ and $12 \%(\mathrm{n}=3)$ are associated with pulmonary embolism. The lower limbs were the most affected $(80.3 \%)$, with predominance on the left side. Bilateral involvement was found in $7 \%$ of cases. Thrombosis was indicative of cancer in $16 \%$ of patients $(n=4)$. While it complicated the development in $84 \%$ of the cases $(\mathrm{n}=21)$. Risk factors for VTE were dominated by females $(56 \%)$, metastases $(64 \%)$, chemotherapy $(80 \%)$, and the presence of a CVC (80\%). Solid tumors were the most frequent $72 \%(\mathrm{n}=18)$ with predominantly digestive cancers $(28 \%)$, pulmonary $(16 \%)$, bladder $(16 \%)$ and breast $(12 \%)$. The most predominant histological type was adenocarcinoma in $44 \%(\mathrm{n}=$ 11). The seven cases of malignant hemopathies are: four non-Hodgkin's lymphomas, two multiple myelomas and one case of chronic myeloid leukemia. The initial curative treatment as a relay used low molecular weight heparin in $92 \%$ of patients $(\mathrm{n}=$ 23). Unfractionated heparin was used in two patients with severe renal failure, the only ones receiving vitamin $\mathrm{K}$ antagonist maintenance therapy. Short-term outcomes were favorable in $92 \%(n=23)$, two deaths Have been attributed to severe pulmonary embolism. Significant progress has been made in the context of cancer-related VTE. Nevertheless, there remains a considerable field of unresolved clinical issues. Numerous studies are underway and may lead to advances not only in the treatment of VTE associated with cancer but also in the treatment of the cancerous disease itself.
\end{abstract}

Keywords: Thrombosis, Cancer, Anticoagulants, Pulmonary Embolism

\section{Introduction}

The association between deep vein thrombosis (DVT) and cancer is a frequent clinical situation described for the first time in 1865 by the French physician Armand Trousseau [1]. Venous thromboembolic disease (VTE) affects 10 to $20 \%$ of cancer patients $[2,3]$ with a very variable incidence depending on the type of cancer and its degree of extension, the metastatic stages being much more thrombogenic than the localized stages. The relationship between thrombosis and cancer is in fact reciprocal; the presence of a cancer multiplies the risk of thrombosis by 4 and up to 6 in the case of associated chemotherapy. The risk of developing cancer in the course of a DVT, in particular unexplained, is very high in the year following an initial episode of thrombosis [2, 4], which raises the question of the interest of Systematic research because of the pejorative nature of this association. VTE disease is the second leading cause of death in cancer [3] and is therefore an unfavorable prognostic factor with decreased in survival [5]. In addition, patients with cancer and VTE have an increased risk of VTE recurrence including optimal treatment and major bleeding complications [6]. 
The physiopathology of thrombosis in a cancer context is complex. The links between mechanisms of coagulation and tumorogenesis are multiple and reciprocal; the tumor cells are procoagulant as well as the conditions related to cancers are prothrombogenic, and the mechanisms of coagulation contribute to the development and the tumor diffusion [7]. In addition to the direct prothrombogenic effects of tumors, we must associate their indirect effects which are related to the patient and those associated with therapeutic approaches. Treatment of VTE in this context is therefore a major issue. Numerous recommendations have been published to guide therapeutic strategies.

The objective of this study is to analyze the epidemiological, clinical diagnosis and therapeutic characteristics of DVT associated with cancers in patients attending the internal medicine-oncology department of the Ibn Sina Military Hospital in Marrakech.

\section{Materials and Methods}

This is a descriptive, retrospective and analytical study over a two-year period from January 2013 to December 2014. All patients with venous thromboembolic disease associated with histologically confirmed cancer were counted. Patients with catheter-related thrombosis were also included.

Were excluded all patients with no histological confirmation of cancer, patients under 18 years of age, unconfirmed imaging VTE, septic thrombosis and patients with unusable medical records.

For each patient, demographic criteria, thromboembolic history, risk factors for VTE, diagnostic methods, thrombosis localization, cancer localization, histological type of cancer and therapeutic strategy were analyzed.

\section{Results}

Among the 158 patients with neoplasia (solid cancer malignant hemopathies), we found 25 cases with venous thrombosis, including 14 women (56\%) and 11 men (44\%) with a sex ratio of Female/Male of 1.27. The average age of our patients at the time of diagnosis was 55.9 years. In males, the average age was 60 years, while for females it was 53.2 years. The incidence of cancer-associated thrombosis was $15.8 \%$ during the study period.

Thrombosis was revealing of cancer in $16 \%$ of patients (n $=4)$ whereas it complicated cancer development in $84 \%(\mathrm{n}=$ 21). The history of DVT is found in only one patient. The thrombosis occurred twelve years before the diagnosis of cancer in a context of immobilization plastered. Solid tumors were the most frequent and accounted for $72 \%(\mathrm{n}=18)$, while hemopathies represented only $28 \%(n=7)$. Among the solid cancers, it is the digestive cancers which predominate found in $28 \%$ followed respectively lung and bladder cancer $16 \%$ each, and the breast cancer found in $12 \%$ of the cases. The most predominant histological type was adenocarcinoma in $44 \%(n=11)$. The seven cases of malignant hemopathies are: four non-Hodgkin's lymphomas, two multiple myelomas and one case of chronic myeloid leukemia, respectively $8 \%$, $6 \%$ and $5 \%$.

Risk factors for VTE in cancer patients were dominated by females (56\%), advanced age over 65 years (52\%), metastasis (64\%) and chemotherapy (80\%) and the presence of a CVC in $80 \%$.

Thrombosis is confirmed by two types of methods: venous echo-doppler in 21 patients and angio-scanner in eight patients. The distribution of venous thrombosis is dominated by the veins of the lower limbs, which represent the most frequent localization( $64 \%$ of cases), nine in the lower left limb $(36 \%)$, four in the lower right limb $(16 \%)$ and three bilateral $(12 \%)$. Other locations are less frequent; two cases of upper limb DVT (8\%), one portal thrombosis (4\%), three cases of CVC thrombosis (12\%) and three cases of pulmonary embolism secondary to DVT (12\%).

All patients received anticoagulant therapy according to international guidelines. $92 \%$ of them $(n=23)$ were treated with low molecular weight heparin (LMWH), Tinzaparin in eighteen patients and Enoxaparin in four patients). Unfractionated heparin (UFH) was used in two patients because of severe renal insufficiency (GFR $<25 \mathrm{ml} / \mathrm{min}$ ) contraindicating the use of LMWH. The use of vitamin K antagonist (VKA) is limited to patients who can not receive heparin therapy with LMWH. Both patients had advanced renal failure, the molecule used was acenocoumarol. LMWH was prescribed in all patients for a minimum duration of 3 months, and was continued for a variable duration ranging from 11 days to 9 months. With regard to catheter-related DVT, the duration was six weeks for the case where the catheter was removed and 3 months for the other two. Elastic restraint was systematically prescribed in all our patients. Early lifting was always the rule.

The short-term outcome was favorable in $84 \%$ of patients $(\mathrm{n}=21)$, with regression of clinical signs related to thrombosis. There was no recurrence under treatment or bleeding complications. But the evolution was marked by the appearance of three cases of pulmonary embolisms of which two were fatal.

\section{Discussion}

Described for the first time by Armand Trousseau, the association of venous thromboembolic disease and cancer has been widely validated in clinical practice. Venous thrombosis may reveal cancer, but cancer and its treatments are also risk factors for VTE $[8,9]$. The characteristics of the thrombosis-associated cancer population are close to those reported in the literature, Whether for the average age between 66 and 69 years, or the slight predominance of females $[10,11]$. VTE disease affects 10 to $20 \%$ of cancer patients $[2,3]$, with a very variable incidence according to the type of cancer considered and its degree of extension, the metastatic stages being much more thrombogenic than the localized stages $[2,3,12]$. 18 to $34 \%$ of patients with VTE have cancer known or discovered at the time of diagnosis of 
thrombosis $[10,13,14,15] .4$ to $10 \%$ of patients with idiopathic thrombosis develop cancer within 2 years of diagnosis of VTE $[9,13,16,17]$. This figure increases to $20 \%$ in the case of recurrent idiopathic DVT, migratory or bilateral [10, 18, 19]. VTE disease may be the first manifestation of otherwise completely silent cancer. Some venous thrombosis may appear suspicious by their atypical localization, their character (spontaneous, recurrent, migratory, blue phlebitis...) or by their echographic appearance (suspended thrombosis, anechogenic...) [20]. In this series, DVT revealed cancer in $15.8 \%$ of cases, which is roughly consistent with the literature.

Several factors may contribute to the increased risk of thrombosis in cancer patients (Table 1). The incidence of thrombosis is higher in the first few months after the diagnosis of cancer. VTE is particularly common in the most aggressive and advanced cancers [21, 22, 23, 24].

Table 1. Different risk factors for DVT in cancer patients [25, 26, 27, 28].

\begin{tabular}{lll}
\hline Patient Factors & Cancer Factors & Treatment Factors \\
\hline - age $>65$ years & - histological type & - hospitalization \\
- Co-morbidities (hepatic, renal, cardiac, & - localization: pancreas, stomach, kidney, & - surgery \\
pulmonary, obesity, infection) & gynecological, lymphoma, lung, bladder, testis & - chemotherapy \\
- history of VTE & - recent diagnosis $<3$ to 6 months & - hormone therapy \\
- thrombocytosis & & - Treatment with angiogenesis inhibitors \\
- hyperleukocytosis & & - growth factors \\
- anemia & & - central venous catheter; in this case thrombosis \\
& depends on: \\
& & - number of insertion attempts, \\
& & - type of cancer, \\
- immobility, Low performance status & - the previous CVC insertion, & - type of catheter used, \\
& & - the technique of insertion, \\
\end{tabular}

Clinical signs leading to the diagnosis of DVT are dominated by edema, pain, collateral venous circulation and Calf pain on dorsiflexion of the foot in the presence of thrombosis in the lower limb [29]. Ultrasound coupled with color Doppler is the method of choice for the diagnosis of DVT and is the first-line examination in the diagnostic strategy. It is easily accessible, fast, inexpensive and simple, non-invasive and non-irradiating. Its performance is excellent with a sensitivity of $100 \%$ and a specificity of $93 \%$ [30]. The existence of a DVT is demonstrated by the ultrasound discovery of the non-compressible character of the vein, associated with a dilation of the vein and/or direct visualization of the thrombus. The compressible or noncompressible character of the vein is correlated with the presence of a thrombus with a sensitivity of $96.3 \%$ and a specificity of $93.5 \%$, the visualization of the thrombus thanks to the color doppler has a sensitivity of $100 \%$ and a specificity of $93.3 \%$ [30]. The exploration of the ilio-cava and even femoro-popliteal axes can be done by angioscanner at the same time as the exploration of the pulmonary arteries. The diagnosis is based on evidence of a central or adherent intra-luminal defect associated with venous enlargement. In case of sequelae of thrombosis, the veins are of small size with a thickened wall with a heterogeneous enhancement or even calcifications. It requires, however, good kidney function, its sensitivity is $100 \%$ and its specificity is $96 \%$. In this series, the angioscanner was performed in eight patients, five cases in favor of an isolated DVT, while in three patients it showed a DVT associated with pulmonary embolism.

Treatment of venous thromboembolic disease is particularly difficult to manage in patients with cancer. Under this circumstance, conventional anticoagulant therapy that combines a short period of heparin (UFH or LMWH) with VKA is less effective and less well tolerated than in cancerfree patients. The risk of recurrent thromboembolism is 3 times greater and that of major bleeding is 6 times greater in cancer patients despite well-conducted VKA treatment [13, $21,31]$. Several studies have prospectively compared the early relay by VKA and the progression of long-term low molecular weight heparin in cancer patients [32, 33, 34, 35]. The meta-analysis of these studies shows that LMWHs significantly reduce thromboembolic recurrences without increasing bleeding or altering mortality [36]. These results led various groups of experts to recommend the use of LMWH (Dalteparin, Enoxaparin or Tinzaparin) for 3 to 6 months in patients with cancer and VTE [21, 37, 38, 39, 40]. In this series, the duration of treatment in 23 patients was between 3 months and 6 months. The patient, who has hepatocellular carcinoma with portal vein thrombosis, was treated for 9 months, which is consistent with literature and the national recommendations.

Beyond six months, in the absence of a specific study, the continuation of LMWH rather than the passage to VKA will be discussed on a case-by-case basis, depending on the severity of the VTE and the cancerous disease. However, it is suggested that anticoagulant treatment with prophylactic dose of LMWH be continued for as long as the cancer is active or undergoing treatment. In the event of recurrence of thrombosis under well-conducted AVK, long-term treatment with LMWH is recommended. If recurrence occurred under 
LMWH, prospective series results suggest a $20 \%$ increase in LMWH dose $[13,41]$. In case of failure, the installation of a filter of the vena cava should be considered. If the filter is placed for recurrence; anticoagulant therapy should be continued. If the filter is placed for a contraindication; when this disappears, the anticoagulant treatment must be resumed.

Platelet monitoring is no longer recommended in cancer patients under LMWH in the medical context. Biweekly monitoring of platelet count is only required in special cases (post-surgical situation, history of heparin treatment in the last 3 months, presence of co-morbidities). It is advisable to reduce the dose of LMWH by $50 \%$ when the platelet count is $<50 \times 10^{9} / \mathrm{L}$ and to discontinue treatment when it is $<30 \times$ $10^{9} / \mathrm{L}[13,42]$.

In the particular case of central venous catheter (CVC) thrombosis, the curative treatment of patients with cancer involves LMWH for at least 3 to 6 months or more in the presence of risk factors for recurrence [43]. The maintenance of the catheter requires that it is essential, functional, well positioned and uninfected, with a favorable clinical course. In this case, anticoagulant therapy should be continued as long as a catheter is in place. The primary prevention of catheter thrombosis is based on a positioning of its distal end at the junction of the superior vena cava and the right atrium because the main risk factor for thrombosis after CVC placement is the malposition of its distal end. Systematic prevention with anticoagulants is not recommended in this indication $[9,44,45]$.

Direct antagonists of factor IIa and factor Xa, usually referred to as direct oral anticoagulants (DOAC), have been compared to AVK in several studies. These studies make it possible to affirm the non-inferiority of the new molecules in terms of efficiency and their superiority in terms of safety $[13,46,47]$. Direct comparisons between DOAC and LMWH in patients with active cancer are ongoing. In the future, DOACs could be a solution of choice because of their simplicity and safety of use, despite the possibility of interactions not inconsiderable in particular with the targeted therapies that share with them certain pathways of metabolism and transport [21, 46, 48, 49].

In everyday practice, the therapeutic decision is often difficult, especially in advanced polymetastatic stages or contraindications to anticoagulants. Awareness is needed in order to allow the implementation of programs to improve the therapeutic management of VTE in patients with cancer. This requires multidisciplinary reflection and the establishment of an observational register of VTE in these patients. This registry will promote adherence to recommendations, the establishment of a multidisciplinary consultation meeting and the creation of a common database useful for clinical research in thrombosis and cancer [50, 51].

\section{Conclusion}

More than 150 years after its first description, the relationship between thrombosis and cancer is still current, as is its pejorative character. Significant progress has been made in the screening of occult cancer in case of unexplained and particularly unusual thrombosis, in primary prevention and in the management of thrombosis in patients with cancer. Nevertheless, there remains a considerable field of unresolved clinical questions, in particular in the most valid strategy for the systematic investigation of occult cancer, the modalities of management of thromboembolic recurrences under anticoagulant and of patients with severe thrombocytopenia, the monitoring and adjustment of LMWH doses in case of renal failure, the role of anticoagulants in slowing tumor progression and in improving the survival of these patients, and in clarifying the role of oral anticoagulants in prevention and treatment of thrombosis associated with cancer.

Numerous studies are underway and may lead to advances not only in the treatment of VTE associated with cancer but also in the treatment of the cancerous disease itself.

\section{References}

[1] AA. Khorana. Malignancy, Thrombosis and Trousseau: the case for an eponym. Journal of Thrombosis and Haemostasis. 2003; (1): 2463-65.

[2] R. Belhadj Chaidi, et al. Application des recommandations dans le traitement de la maladie thromboembolique veineuse chez les patients atteints de cancer: étude rétrospective sur 145 cas. Journal des Maladies Vasculaires (2013) 38, 185-192.

[3] HK. Chew, T. Wun, D. Harvey, H. Zhou, RH. White. Incidence of venous thromboembolism and its effect on survival among patients with common cancers. Arch Intern Med 2006; 166: 458-64.

[4] JT. Murchison, L. Wylie, DL. Stockton. Excess risk of cancer in patients with primary venous thromboembolism: a national, population-based cohort study. Br J Cancer 2004; 91: 92-5.

[5] T. Sorensen, L. Mellemkjaer, J. Olsen, et al. Prognosis of cancers associated with venous thromboembolism. N Engl J Med 2000; 343: 1846-50.

[6] D. Farge, P. Debourdeau, M. Beckers, et al. International clinical practice guidelines for the treatment and prophylaxis of venous thromboembolism in patients with cancer. J Thromb Haemost 2013; 11: 56-70.

[7] J. Médioni, JP. Guastalla, L. Drouet. Thrombose veineuse et cancer du sein. Bull Cancer 2012; 99: 199-210.

[8] HR. Buller, et al. Cancer and thrombosis: from molecular mechanisms to clinical presentations. J Thromb Haemost 2007; 5 (Suppl. 1): 246-54.

[9] P. Girard, C. Raynaud, G. Meyer, F. Parent, B. Besse. Thrombose et cancer bronchique. Revue de Pneumologie clinique (2008) 64, 85-91.

[10] A. Bertoïa, M.-T. Barrellier, V. NGuyen-Van, L. Berger, C. Le Hello. Incidence et distribution de 1026 thromboses veineuses des membres inférieurs diagnostiquées par écho-Doppler chez 3263 patients atteints de cancer. Journal des Maladies Vasculaires (2013) 38, 243-251.

[11] AA. Khorana, et al. Risk factors for chemotherapy-associated venous thromboembolism in a prospective observational study. Cancer 2005; 104 (12): 2822. 
[12] T. Wun, RH. White. Epidemiology of cancer-related venous thromboembolism. Best Pract Res Clin Haematol 2009; 22: 923.

[13] G. Meyer, P. Girard. Maladie veineuse thromboembolique et cancer. Revue des Maladies Respiratoires Actualités (2016) 8, 489-496.

[14] C. Martinez, AT. Cohen, L. Bamber, S. Rietbrock. Epidemiology of first and recurrent venous thromboembolism: a population-based cohort study in patients without active cancer. Thrombosis and Haemostasis 112. 2/2014.

[15] M. Cushman, et al. Deep vein thrombosis and pulmonary embolism in two cohorts: the longitudinal investigation of thromboembolism etiology. Am J Med 2004; 117: 19-25.

[16] M. Carrier, et al. Systematic review: the Trousseau syndrome revisited: should we screen extensively for cancer in patients with venous thromboembolism? Ann Intern Med 2008; 149: 323-33.

[17] C. Ay, D. Dunkler, C. Marosi, et al. Prediction of venous thromboembolism in cancer patients. Blood 2010; 116: 537782.

[18] C. Boccalon, P. Leger, D. Barcat, J. Guilloux, H. Boccalon Thromboses veineuses des membres inférieurs et de la veine cave inférieure. EMC. Cardiologie. 11-730-A-10 (2004); 11p.

[19] C.-M. Samama et al. Prévention de la maladie thromboembolique veineuse périopératoire et obstétricale. Annales Françaises d'Anesthésie et de Réanimation 24 (2005) 853-861.

[20] M. Pavic, P. Debourdeau, M. Aletti, D. Farge-Bancel, H Rousset. Maladie veineuse thromboembolique et cancer. Rev Med interne. 2006; (27): 313-322.

[21] HE. Fuentes, AJ. Tafur, JA. Caprini. Cancer-associated thrombosis. Disease-a-Month 62 (2016), 5, 121-158.

[22] AY. Lee, MN. Levine, G. Butler, et al. Incidence, risk factors, and outcomes of catheter-related thrombosis in adult patients with cancer. J Clin Oncol. 2006; 24 (9): 1404-1408.

[23] M.-A. Sevestre, et al. Compliance with recommendations of clinical practice in the management of venous thromboembolism in cancer: The CARMEN study. J Mal Vasc39, 3, 2014, 161-168.

[24] GT. Gerotziafas, I. Elalamy. Risque thromboembolique en oncologie: réalités, actualités et perspectives. Bull Cancer 103, 9, 2016, 764-775.

[25] AA. Khorana, GC. Connolly. Assessing Risk of Venous Thromboembolism in the patient with cancer. 2009; (27): 4839-47.

[26] L. Drouet. Thromboembolic risk associated with use of angiogenesis inhibitors used for the treatment of cancers. Pathologie-Biologie. 2008; (56): 195-204.

[27] F. Elice. Thrombosis associated with angiogenesis inhibitors. Best Practice and Research Clinical Haematology. 2009; (22): 115-128.

[28] M. Chavez-Macgregor, et al. Complications associated with erythropoietin-stimulating agents in patients with metastatic breast cancer: A Surveillance, Epidemiology, and End Results-Medicare Study. Cancer 2011; 117: 3641-9.
[29] I. Marie, H. Levesuque, N. Cailleux, E. Primard, et al. Les thromboses veineuses profondes des membres. A propos de 49 cas. Rev Méd Interne. 1998; (19): 399-408.

[30] P. Prandoni, P. Polistena, et al. Upper-extremity deep vein thrombosis. Risk factors, diagnosis, and complications. Arch Intern Med. 1997; (157): 57-62.

[31] P. Prandoni, AW. Lensing, et al. Recurrent venous thromboembolism and bleeding complications during anticoagulant treatment in patients with cancer and venous thrombosis. Blood 2002; 100: 3484-8.

[32] SR. Deitcher, CM. Kessler, et al. Secondary prevention of venous thromboembolic events in patients with active cancer: enoxaparin alone versus initial enoxaparin followed by warfarin for a 180 day period. Clin Applied Thromb Hemost 2006; (12): 389-96.

[33] RD. Hull, GF. Pineo, et al. Long term low-molecular-weight heparin versus usual care in proximal vein thrombosis patients with cancer. Am J Med 2006; (119): 1062-72.

[34] AY. Lee, MN. Levine, RI. Baker, et al. Low-molecular-weight heparin versus a coumarin for the prevention of recurrent venous thromboembolism in patients with cancer. $\mathrm{N}$ Engl J Med 2003; (349): 146-53.

[35] G. Meyer, Z. Marjanovic, et al. comparison of Low-molecularweight heparin and warfarin for the secondary prevention of venous thromboembolism in patients with cancer: a randomized controlled study. Arch Intern Med 2002; (162): 1729-35.

[36] ML. Louzada, H. Majeed, PS. Wells. Efficacy of Lowmolecular-weight heparin versus vitamin $\mathrm{K}$ antagonists for long term treatment of cancer-associated venous thromboembolism in adults: a systematic review of randomized controlled trials. Thromb Res 2009; (123): 837-44.

[37] P. Mismetti, JM. Baud, F. Becker, et al. Guidelines for good clinical practice: prevention and treatment of venous thromboembolism in medical patients. J Mal Vasc2010; (35): 127-136.

[38] D. Farge, L. Bosquet, D. Kassab-Chahmi, et al. 2008 French national guidelines for the treatment of venous thromboembolism in patients with cancer: report from the working group. Crit Rev Oncol Hematol 2010; (73): 31-46.

[39] C. Kearon, SR. Kahn, G. Agnelli, et al. Antithrombotic therapy for venous thromboembolic disease: American College of Chest Physicians Evidence-Based Clinical Practice Guidelines (8th Edition). Chest. 2008; (133): 454S-545S.

[40] P. Debourdeau, et al. 2008 SOR guidelines for the prevention and treatment of thrombosis associated with central venous catheters in patients with cancer: report from the working group. Ann Oncol. 2009; (20): 1459-71.

[41] R. Ihaddadene, G. Le Gal, A. Delluc, M. Carrier. Dose escalation of low molecular weight heparin in patients with recurrent cancer-associated thrombosis. Thromb Res 2014; 134: $93-5$

[42] L. Geffray. Actualités en thrombose et cancer. La revue de médecine interne 32 (2011) 265-267.

[43] D. Farge, C. Durant, S. Villiers, et al. G. Lessons from French National Guidelines on the treatment of venous thrombosis and central venous catheter thrombosis in cancer patients. Thromb Res, 2010; 125: 108-16. 
[44] S. Villiers. La pratique et le traitement des thromboses de cathéter au cours du cancer. Journal des Maladies Vasculaires (2015) 40, 284-291.

[45] A. Elias, P. Debourdeau, et al. Traitement curatif de la maladie thromboembolique veineuse et prise en charge des thromboses veineuses sur cathéter chez les patients atteints de cancer. Presse Med. 2009; 38: 220-230.

[46] C. Kearon, EA. Akl, J. Ornelas, A. Blaivas, D. Jimenez, H. Bounameaux, et al. Antithrombotic Therapy for VTE Disease: CHEST Guideline and Expert Panel Report. Chest 2016; 149: 315-52.

[47] Es N. Van, M. Coppens, S. Schulman, S. Middeldorp, HR. Buller. Direct oral anticoagulants compared with vitamin K antagonists for acute venous thromboembolism: evidence from phase 3 trials. Blood 2014; 124: 1968-75.
[48] M. Franchini, et al, Cancer-associated thrombosis: investigating the role of new oral anticoagulants, Thromb Research 135, 5, 2015, 777-781.

[49] S. Noble, J. Sui. The treatment of cancer associated thrombosis: does one size fit all? Who should get LMWH/warfarin/DOACs? Thrombosis Research 140S1 (2016) S154-S159.

[50] S. Noboa, D. Mottier, E. Oger. Estimation of a potentially preventable fraction of venous thromboembolism: a community based prospective study. J thromb Haemost 2006; (4): $2720-2$.

[51] I. Mahe, J. Chidiac. Cancer et récidive thromboembolique veineuse: non-respect des recommandations de traitement. Bull Cancer 2014; 101: 295-301. 